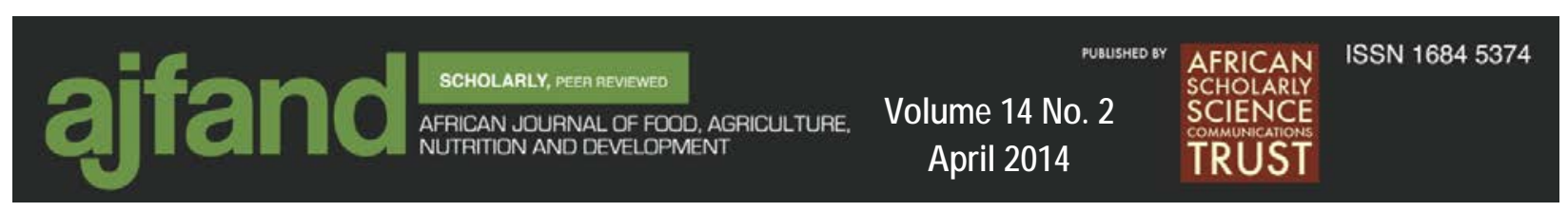

\title{
ACCEPTABILITY OF CHICKEN POWDER IN HOME PREPARED COMPLEMENTARY FOODS FOR CHILDREN IN GHANA
}

\section{Anderson AK ${ }^{1}$, Bediako-Amoa $B^{2}$ and $M$ Steiner-Asiedu ${ }^{2}$}

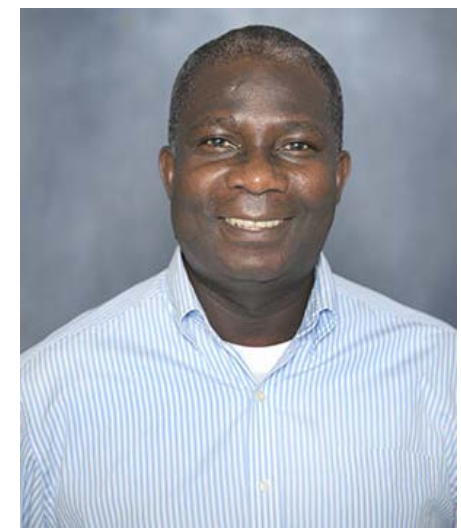

Alex K Anderson

*Corresponding author’s email: fianko@uga.edu

${ }^{1}$ Department of Foods and Nutrition, University of Georgia

${ }^{2}$ Department of Nutrition and Food Science, University of Ghana 


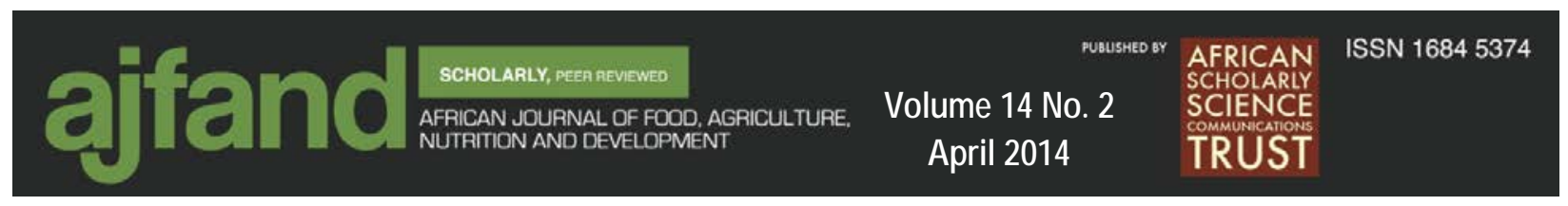

\section{ABSTRACT}

The persistent problem of childhood malnutrition and growth faltering in sub-Saharan African countries such as Ghana despite food aid intervention programs, suggests rethinking of the content and mode of delivery of such food programs. While several food programs and formulations exist for the treatment and management of childhood malnutrition, most are neither culturally appropriate nor easily accessible to families at the household level. There is, therefore, a need for a food product that can be tailored to a particular culture and local food produce which provides the necessary macronutrients and micronutrients in adequate amount and is easy to use at the household level to prevent malnutrition. The aim of this study was to evaluate the acceptability of incorporating chicken powder into the Ghanaian child's everyday meal. Participants in the study included mothers and their pre-school age children. The chicken powder was incorporated into a breakfast meal (“Tom Brown”) and lunch/supper meal (rice and gravy) on weight basis according to predetermined proportions of the raw ingredients. Each meal consisted of a control sample (not containing chicken powder) and 3 test samples containing different amounts of chicken powder (15\%, 20\% and 25\% by weight). Overall, both mothers and children rated both control samples highest compared to their respective test sample for preference. Interestingly, mothers were able to tell the differences in the test samples, but the children could not tell the difference between the test samples by the content of the chicken powder according to the results of the test for homogeneity. The results from the acceptability test as well as individual observation by the research team suggest that chicken powder can be used as animal source protein in the treatment and management of malnutrition by incorporating it into common staple foods. According to findings of this study, parents are more likely to incorporate the chicken powder (an animal source quality protein) in their children's diet, if not for the entire family. The incorporation of chicken powder into complementary foods will improve the nutritional value, especially the protein and micronutrient content.

Key words: Chicken, Acceptability, Ghana, Malnutrition, Feeding 


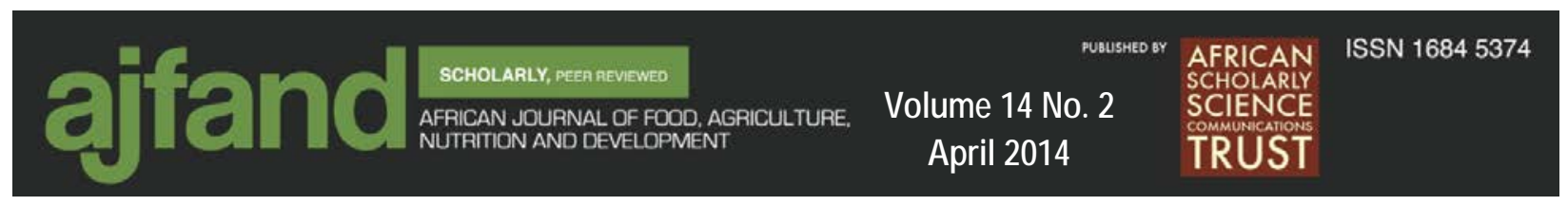

\section{INTRODUCTION}

Childhood malnutrition continues to be a burden in developing countries, particularly in subSaharan Africa [1]. In sub-Saharan Africa, approximately $42 \%$ of preschool children are stunted [1]. Mokhtar et al. [2] have also reported persistent undernutrition mostly in sub-Saharan African countries including Ghana. de Onis and Blossner [3] have reported undernutrition among preschool children to be a significant public health problem, while the Food and Agricultural Organization (FAO) [4] has observed that micronutrient deficiencies, especially iron, zinc, iodine and vitamin A are unusually high within this region of the world. The FAO report suggests that $76.5 \%$ of preschool children are anemic, while $10.0 \%$ die from micronutrient deficiency and associated illnesses. To combat the problem of under nutrition in Sub-Saharan Africa and other developing countries around the world, humanitarian food aid has typically been distributed to vulnerable groups in these countries who are at greatest risk for malnutrition. In most cases, the affected groups have used this humanitarian food aid as a replacement instead of as a supplement to their inadequate and less nutritious diet. Other programs have also used fortified food-aid commodities as a means of improving the nutritional content and inadequacy of the diet of children in emergency as well as in extreme hunger.

To this end, a number of formulations have either been proposed or tested with the hope of using them to prevent or manage malnutrition among children, especially in developing countries, with little success [3-7]. This is because most of these programs have operated within the confines of an institution or a particular setting without the direct involvement of families/households in the preparation of the therapeutic food as well as the treatment or management of childhood malnutrition. The continued increase in childhood malnutrition in sub-Saharan Africa suggests behavioral, cultural and environmental factors may be driving this public health problem. So far, most intervention and supplementation programs have not been sustainable or routinely incorporated into the child's diet at the household level because of cost, acceptability and availability of the fortificant in the local communities and households. There is, therefore, a need for a fortificant which is cheap, acceptable and easily incorporated into the child's diet at the household level by the primary care-giver without the potential for toxicity. Chicken powder (CP) may be able to be used as a fortificant and provide a source of animal protein to a child's diet. Apart from being cheap, CP is a source of quality animal protein and essential micronutrients. $\mathrm{CP}$ also holds the potential of eliminating or reducing malnutrition in lowincome communities within developing countries when incorporated into regularly consumed home prepared meals as well as be used in emergency food aid. Chicken powder provides health benefits in addition to quality protein, fatty acids and essential micronutrients (Table 1). The protein content of CP (72-75\%) exceeds both that of Corn Soy Blend (CSB; 17\%) and Defatted Soy Flour (DSF; 50\%) by weight. For example, the poor digestibility of protein, if any, in the diets of children from developing countries is due to the use of cereals and pulses such as beans and lentils as major sources of protein (Report of the Joint FAO/WHO Expert Consultation 1990). This makes CP an important ingredient in the diet of preschool children, who because of their rapid growth and small stomach volume, need quality protein and other essential nutrients 


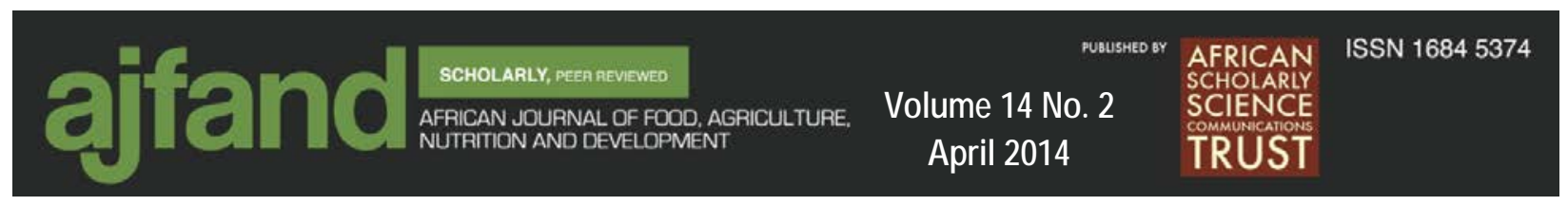

to prevent malnutrition, particularly protein-energy malnutrition and stunting. Again, in underserved areas where high quality protein is not available or limited because of cost, CP would serve as an excellent supplement to local diets. Chicken, unlike beans and grains, contains no phytates that interfere with mineral absorption.

\section{Objectives}

The purpose of this study was to assess the acceptability of incorporating CP in the child's diet at the household level. Data from this study could subsequently be used to design an intervention to examine the impact of adding CP in the child's diet on the growth, development and nutritional status of undernourished children.

\section{MATERIALS AND METHODS}

\section{Study design and population}

This acceptability study was carried out in December 2009 among mothers and children in a farming community (Otinibi) in the Greater Accra Region of Ghana. Measured quantities of CP were added to a breakfast meal (Tom Brown Porridge) and lunch/dinner meal (rice and stew/gravy), which were respectively served to mothers and children on two different days. A convenient sample of 53 mothers aged $18-40$ years and 58 children aged $2-5$ years participated in this study. The convenient sample was made up of mother-child pairs, residents of Otinibi who have previously participated in research projects conducted by the research team in the community and were accessible to the researchers. Each meal consisted of four different samples containing different amounts of CP to provide about 20 grams of protein according to the World Health Organization recommendation for pre-school children. Mothers and children were served the four samples of each meal to taste and rate the liking separately. They were also asked to provide comments on each sample. For the younger children, research assistants used a scale consisting of different facial expressions to score the level of acceptance of the sample depending on the child's facial expression after tasting each sample.

\section{Breakfast Meal}

The breakfast meal used in this study was a porridge locally called “Tom Brown”. This porridge is consumed by the majority of households in the study community as well as in most of Ghanaian households. The porridge is usually made from roasted white maize (corn), mixed/dissolved in water, added salt, boiled till some degree of gelling and served with sugar added. Depending on the household food security or socio-economic status, this may be eaten as is (described) or with milk added, with or without bread. For the study, four different porridges prepared were made up of 1 part maize-chicken blend and 9 parts water. The porridges were made with roasted white maize and chicken powder in the following proportions:

1. $100 \%$ roasted maize by weight (no chicken powder)-control

2. $85 \%$ roasted maize by weight $+15 \%$ chicken powder by weight

3. $80 \%$ roasted maize by weight $+20 \%$ chicken powder by weight

4. $75 \%$ roasted maize by weight $+25 \%$ chicken powder by weight 


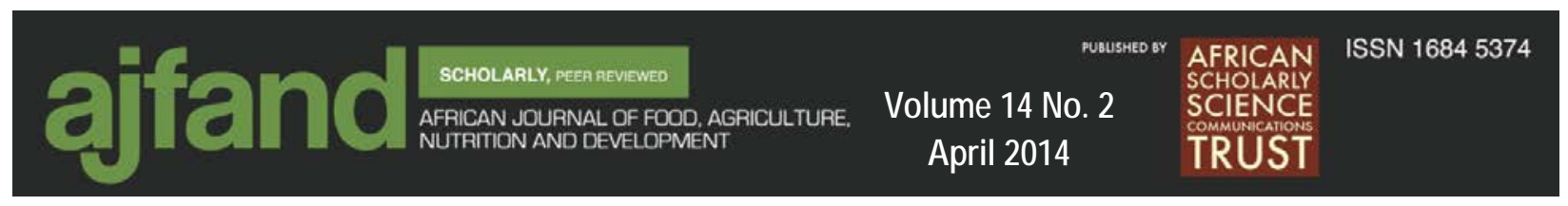

Two teaspoons of ordinary table salt $(\mathrm{NaCl})$ and $200 \mathrm{~g}$ of sugar were added to each batch of mixture, stirred until boiling and allowed to boil under medium heat for 10 minutes.

\section{Lunch/Supper Meal}

For the lunch/supper meal for the study, we used white steamed rice with gravy. Regular steamed white rice was used, with four different gravies containing different amounts of the chicken powder. The gravy was prepared from tomatoes, peppers, onions, salt, vegetable oil and containing no CP (control sample), 15\% CP, 20\% CP and 25\% CP.

\section{Presentation, Tasting and Rating of Samples}

Each food item consisted of four samples served into different coded cups (for the porridge) and plates (for the rice and gravy). The porridge was served on the first day of the study while the rice and gravy was served the following day. On the first study day, a field research assistant presented a tray holding the four porridge samples in cups and spoons to mothers and children (both male and female) to be tasted and rated separately. The rice and gravy was presented in a similar manner the following day but coded differently from the porridge. Participants tasted each of the four samples of the day and provided their ratings for likeness. Between tasting of samples, participants were given water to rinse their mouth after tasting in order to avoid carryover of sensory properties like taste and flavor from the previous sample. Each sample was rated on a 5-point scale (dislike very much [1]; dislike moderately [2]; neither like nor dislike [3]; like moderately [4]; like very much [5]).

The study was approved by the Institutional Review Board of the University of Georgia, and all mothers provided oral consent on behalf of their children and on their own behalf before participating in the study.

\section{Statistical Analysis}

Data was entered and analyzed using SPSS for Windows (version 17.0). Analysis of Variance (ANOVA) and paired sample t-test were used to examine the differences in sensory characteristics between samples within the breakfast meal or lunch/supper meal according to participants rating for likeness. A p-value of $<0.05$ was set as the criterion for statistical significance.

\section{RESULTS}

\section{Ratings of the preference for the breakfast porridge}

For the breakfast porridge, no significant difference in preference of test samples among both mothers and children between samples was observed, although the control sample (not containing any chicken powder) had the highest average score. The preference as well as the overall ratings was very similar among mothers and children (Table 2). Besides the control breakfast meal receiving the highest average score for preference, the study participants 


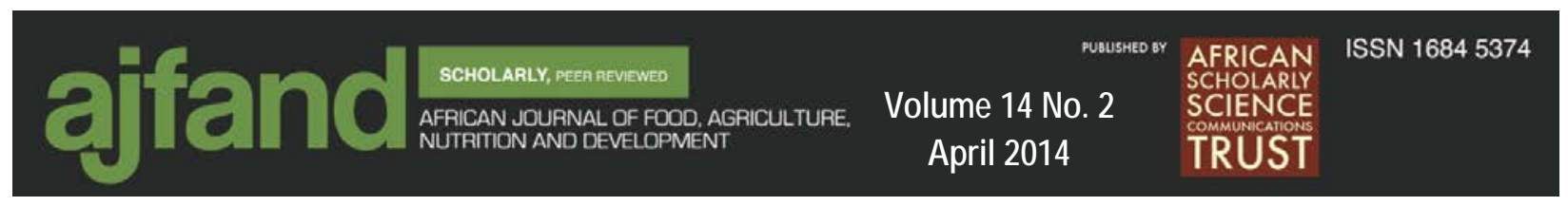

preferred the test samples in this order: porridge containing $25 \% \mathrm{CP}$ by weight and $75 \%$ roasted corn meal by weight, followed by porridge containing $15 \%$ CP by weight and $85 \%$ roasted corn meal by weight and finally porridge containing $20 \%$ CP by weight and $80 \%$ roasted corn meal by weight.

Comparing the preference between the control and test samples, no statistically significant differences in the acceptability among the mothers were observed, although the average preference score for the control sample was highest compared to the 3 test samples. For the children we observed a statistically significant difference in preference between the control sample and the sample containing $20 \%$ CP by weight and $80 \%$ corn meal by weight $(\mathrm{p}<0.05)$, but not any of the other samples or between test samples. Overall, the sample containing 20\% CP was rated poorly by both children and mothers. The score indicates that the children liked or preferred the control sample to all the test samples with the same trend observed among the mothers, although that was not statistically significant.

\section{Ratings of the preference for the rice and gravy}

Both mothers and children scored the control sample (rice and gravy without added chicken powder) the highest on preference compared to the three test samples as in the case of the breakfast porridge (Table 3). There was statistically significant difference between the mean score for preference for mothers $(p=0.01)$ but not the children. Further examination of the preference score using Tukey's HSD post-hoc test showed that mothers strongly preferred the control sample (gravy) over the tests samples. This was obvious in the homogeneity test conducted. The test for homogeneity of the samples showed that mothers were able to distinguish the samples into 2 unique groups, control sample (containing no chicken powder) versus the 3 test samples (containing different levels of dehydrated chicken powder in the gravy) $(p<0.05)$. Another revealing observation reported by mothers is the difference in consistency between the test samples and the control sample with the test gravies being thicker in consistency than the control gravy. The data shows significant difference $(\mathrm{p}<0.05)$ between the mean scores for likeness for samples $0 \%$ vs $20 \%$, $0 \%$ vs $25 \%$, $15 \%$ vs $20 \%, 15 \%$ vs $25 \%$ but not $0 \%$ vs $15 \%$, and $20 \%$ vs $25 \%$ as reported by mothers (Table 3 ). The difference between the control sample versus the test samples containing $20 \%$ and $25 \% \mathrm{CP}$ was attributed to the unusually strong chicken flavor and thicker consistency.

For the children, although the control sample was scored highest on preference compared to the test samples, test for homogeneity did not detect any difference between the control sample and test samples as the case was with the mothers. Again, there were no statistically significant differences between pairs in terms of mean score for preference. This means that even though the children could detect that something had been added to some of the samples, they still liked them unlike their mothers. This is good news for incorporating the chicken powder in their regular diet as a means to improve their intake of quality protein and essential micronutrients. 


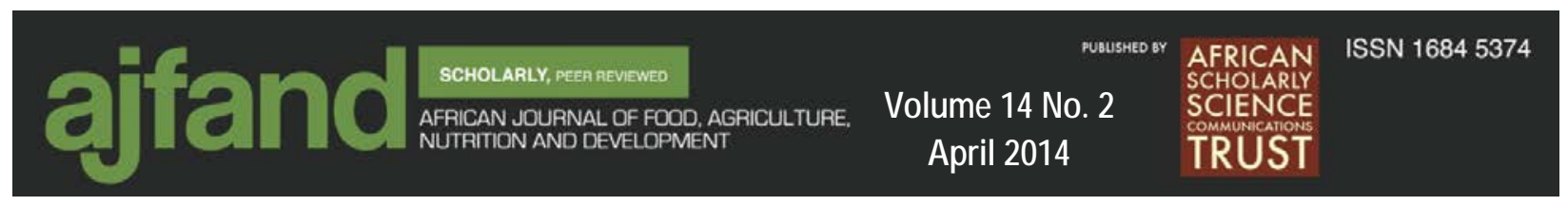

\section{DISCUSSION}

Overall, both mothers and children liked food containing the chicken powder, although they scored the control samples for the porridge and gravy highest over the respective test samples because that is what they are used to. Consistently, the children scored the preference of each of the test samples higher compared to the scoring by the mothers, irrespective of being a porridge or rice and gravy. Mothers who participated in the study said they will definitely incorporate the chicken powder in food to feed their children and the entire family. Mothers enjoyed both the porridge and gravy containing the chicken powder and even requested to be given the left over samples to be used later. A majority of the mothers reported liking the taste, flavor and aroma of the test samples. The children also liked the sample very much as we could tell from their responses and demeanor and facial expressions during the testing and after.

Findings from the current study are similar to what has previously been reported in studies from other developing countries [8,9, 10], although different products were used. In the study by Rowe et al. [10] where the acceptability of corn-soy blend was evaluated in Guatemala, Uganda and Malawi, a majority (74\%) of the respondents said they liked the product because of its sensory characteristics. This observation was also confirmed in the present study, but it is important to mention that the nutritional value of chicken-powder incorporated in porridge or gravy by weight is better than the soy-corn blend $[9,11]$. The protein content of the chicken powder by weight is far higher (72-75\% versus 17\%) compared to the corn-soy blend, although the Protein Digestibility Corrected Amino Acid Score (PDCAAS) values are similar. Also, the iron content of the chicken powder is higher than that of soy concentrate and other legumes generally used in the preparation of complementary foods in child feeding [12]. Moreover, chicken powder contains heme iron which is readily absorbed compared to non-heme iron found in legumes, including soy concentrates [12]. Chicken powder also contains higher amounts of selenium compared to other legumes [12]. This, therefore, makes the chicken powder a superior option for use in the treatment and management of childhood malnutrition such as kwashiorkor.

The higher scoring of both control samples compared to test samples by mothers and children in this study is an indication of participants' familiarity with the control samples (not containing chicken powder). This observation is quite interesting in two ways. First, the participants' ability to score the control sample as the best is because it is what they are used to and have eaten over the years. Even though the control samples were scored higher for preference than the test samples, both mothers and children stated they still liked the porridge with various additions of CP. Second, both mothers and children scored the test sample containing $20 \%$ CP by weight the lowest compared to the other test samples, which was unexpected. It was expected that participants would score the test sample containing 25\% CP by weight the lowest compared to the other test samples. This is because the test sample containing $25 \%$ CP by weight had more chicken flavor and was slightly thicker in consistency compared to the other samples. Assuming the corn flour used for the porridge and the other ingredients for the rice and gravy meal contain negligible protein, about 28 grams of chicken powder (almost the 25\% CP by weight) provides 


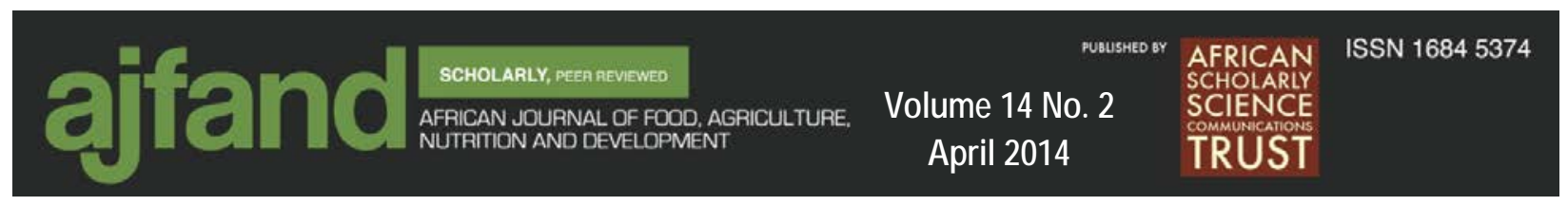

the WHO recommended intake of protein of 20 grams for young children [13]. Test of homogeneity also showed that both mothers and children could not tell the test samples (Tom Brown porridge) contained something different (in this case the chicken powder) from the control sample to which they are accustomed. For the gravy, the test of homogeneity suggested the children could not differentiate between the control and test samples, but the mothers did. This means mothers were able to tell the test samples contained something which was missing from the control sample. Some of the mothers were able to tell the test samples contained chicken whereas the control sample did not because of differences in flavor.

In summary, the results from the acceptability test as well as individual observations by the research team suggest that chicken powder with its high protein quality and essential micronutrient content could be incorporated in common staple foods to improve their quality in young child feeding. According to the findings, parents are more likely to incorporate the chicken powder in their children's diet if not for the entire family. Findings from this study, although promising in improving diet quality for the management and prevention of childhood malnutrition, should be interpreted with caution because of the convenient sample used limiting its generazability.

\section{ACKNOWLEDGEMENT}

The authors are grateful to all mothers and children who participated in the study. We also wish to thank all the research assistants who assisted with the presentation of samples and data collection. We acknowledge the invaluable contribution to the conception of the study by EC Henley of Athens, Georgia, U.S.A.

\section{FUNDING}

The study was supported by funding from the International Dehydrated Foods Inc. MO, USA. 


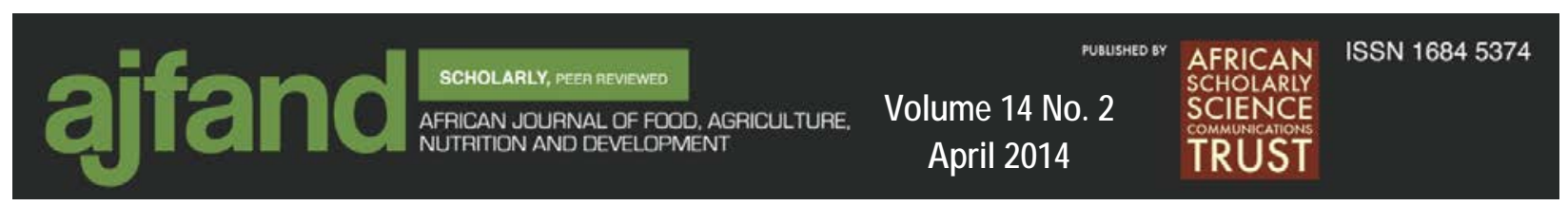

Table 1: Nutrient Content of Dehydrated Chicken Powder per 100 grams*

\begin{tabular}{|c|c|c|}
\hline Nutrient & Unit & Content \\
\hline Water & g & 605 \\
\hline Energy & kcal & 380 \\
\hline Protein & g & $72-75$ \\
\hline PDCAAS & & 1.0 \\
\hline Total Lipid & g & 18 \\
\hline Carbohydrate & g & 0.0 \\
\hline Fiber & g & N/A \\
\hline Ash & g & 5 \\
\hline Calcium & $\mathrm{mg}$ & 1.03 \\
\hline Iron & $\mathrm{mg}$ & 12.7 \\
\hline Magnesium & $\mathrm{mg}$ & 0.085 \\
\hline Phosphorus & $\mathrm{mg}$ & 0.92 \\
\hline Potassium & $\mathrm{mg}$ & 0.38 \\
\hline Sodium & $\mathrm{mg}$ & 0.26 \\
\hline Zinc & $\mathrm{mg}$ & 7.8 \\
\hline Copper & $\mathrm{mg}$ & 0.247 \\
\hline Manganese & $\mathrm{mg}$ & 0.225 \\
\hline Selenium & mcg & 80 \\
\hline
\end{tabular}

*Source of Analysis: Food Ingredients Technology Company, LLC. Springfield, MO

PDCAAS:Protein Digestibility Corrected Amino Acid Score 


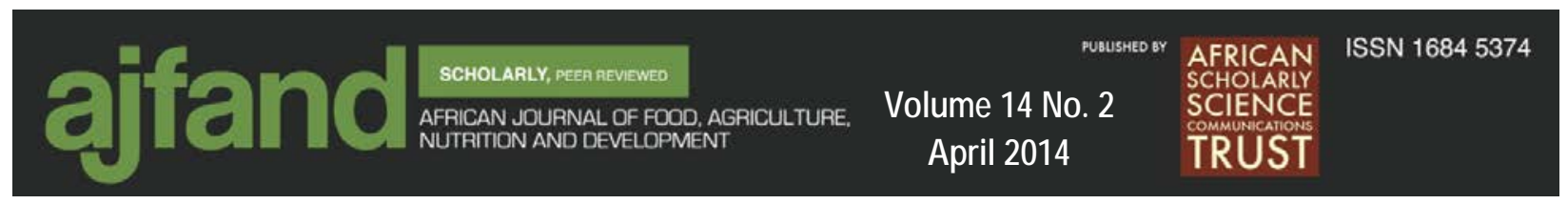

Table 2: Scoring for the Breakfast Meal (Tom Brown Porridge)

\begin{tabular}{|l|c|c|}
\hline Sample & Mothers & Children \\
\hline Porridge contains no DCP (Control ) & $4.38 \pm 1.10$ & $4.67 \pm 0.51$ \\
\hline Porridge contains 15\% DCP by weight & $4.25 \pm 1.18$ & $4.33 \pm 1.19$ \\
\hline Contains 20\% of DCP by weight & $4.13 \pm 1.16$ & $4.21 \pm 1.23$ \\
\hline Porridge contains 25\% of DCP by weight & $4.32 \pm 1.17$ & $4.38 \pm 1.01$ \\
\hline
\end{tabular}

Each product was rated on a 5-point scale (dislike very much [1]; dislike moderately [2]; neither like nor dislike [3]; like moderately [4]; like very much])

Table 3: Scoring for the Lunch/Supper Meal (Rice and Gravy)

\begin{tabular}{|l|c|c|}
\hline \multicolumn{1}{|c|}{ Sample Code } & Mothers & Children \\
\hline Gravy Contains no DCP (Control ) & $4.73 \pm 0.71$ & $4.86 \pm 0.35$ \\
\hline Gravy contains 15\% DCP by weight & $4.71 \pm 0.71$ & $4.78 \pm 0.54$ \\
\hline Gravy contains 20\% DCP by weight & $4.27 \pm 1.07$ & $4.76 \pm 0.65$ \\
\hline Gravy contains 25\% DCP by weight & $4.25 \pm 1.21$ & $4.82 \pm 0.52$ \\
\hline
\end{tabular}

Each product was rated on a 5-point scale (dislike very much [1]; dislike moderately [2]; neither like nor dislike [3]; like moderately [4]; like very much]) 


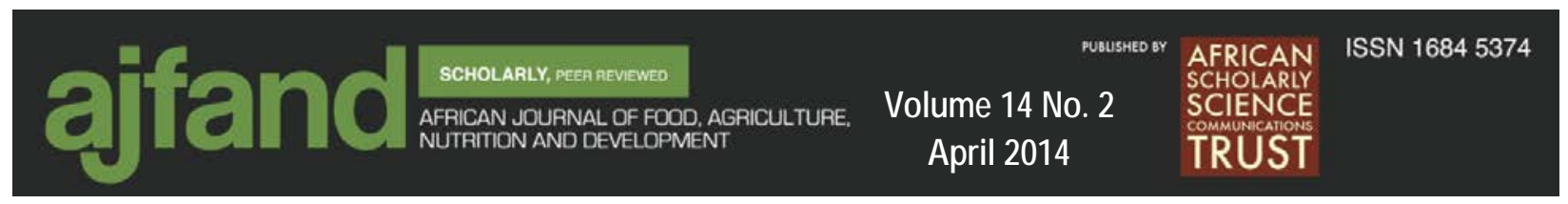

\section{REFERENCES}

1. Mamabolo RL, Alberts M, Steyn NP, Delemarre-van de Waal HA and NS Levitt Prevalence and determinants of stunting and overweight in 3-year-old black South African children residing in the Central Region of Limpopo Province, South Africa. Public Health Nutrition 2005; 8: 501-508.

2. Mokhtar N, Elati J, Chabir R, Bour A, Elkari K and NP Schlossman Diet culture and obesity in northern Africa. J Nutr 2001; 131: 887S-892S

3. de Onis $\mathbf{M}$ and $\mathbf{M}$ Blossner Prevalence and trends of overweight among preschool children in developing countries. Am J Clin Nutr 2000; 72: 1032-1039.

4. Food and Agriculture Organization Anti-hunger programme A twin-track approach to hunger reduction: priorities for national and international action. Food and Agriculture Organization of the United Nations November 2003.

5. Hoppe C, Molgaard C and KF Michaelsen Cow's milk and linear growth in industrialized and developing countries. Annu Rev Nutr 2006; 26: 131-73.

6. Golden MH Proposed recommended nutrient densities for moderately malnourished children. Food and Nutr Bull. 2009; 30(3): S267-S342.

7. Michaelsen KF, Hoppe C, Roos N, Kaestel P, Stougaard M, Lauritzen L, Molgaard C, Girma $\mathbf{T}$ and $\mathbf{H}$ Friis Choice of foods and ingredients for moderately malnourished children 6 months to 5 years of age. Food Nutr Bull. 2009; 30(3): S343-S404.

8. Hoppe C, Andersen GS, Jacobsen S, Molgaard C, Friis H, Sangild PT and KF Michaelsen The use of whey or skimmed milk powder in fortified blended foods for vulnerable groups. J Nutr. 2008; 138(1): 145S-161S.

9. Hotz C and RS Gibson Traditional food-processing and preparation practices to enhance the bioavailability of micronutrients in plant-based diets. J Nutr. 2007; 137: 1097-1100.

10. Rowe JP, Brodegard WC, Pike OA, Steele FM and ML Dunn Storage, preparation, and usage of fortified food aid among Guatemalan, Ugandan, and Malawian beneficiaries: A field study report. Food Nutr Bull. 2008; 29(3): 213-220.

11. de Pee S and MW Bloem Current and potential role of specially formulated foods and food supplements for preventing malnutrition among 6-23 months old and treating moderate malnutrition among 6-59 months old children. In, WHO, UNICEF, WFP and UNHCR Consultation on the Dietary Management of Moderate Malnutrition in Under-5 Children by the Health Sector, World Food Program, 2008; 1-40. 


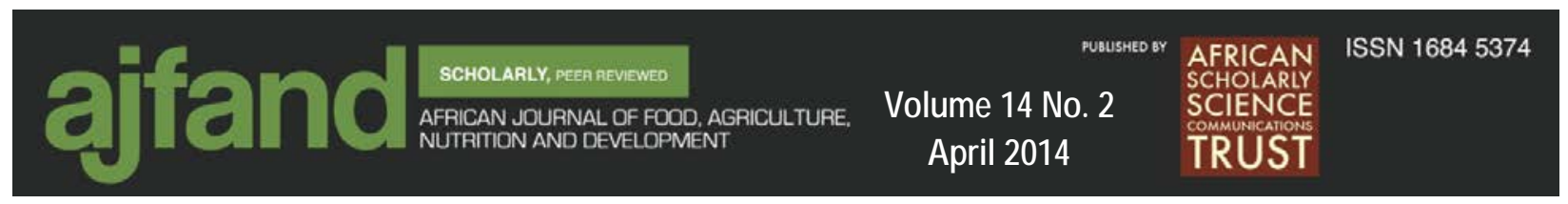

12. Protein Quality Evaluation, Report of the Joint FAO/WHO Expert Consultation. Rome, Italy. Food and Agriculture Organization and World Health Organization. Food and Agriculture Organization of the United Nations; 1990.

13. WHO Technical Report Series Protein and amino acid requirements in human nutrition. Report of a Joint WHO/FAO/UNU Expert Consultation, United Nations University 